\title{
Urheberrecht
}

Rainer Kuhlen*

\section{Zum Vorschlag der EU-Kommission für eine neue Urheberrechtsrichtlinie}

\author{
Die ökonomische Verwertungssicht dominiert weiter gegenüber den offenen Austauschformen \\ in Bildung und Wissenschaft
}

DOI 10.1515/iwp-2017-0026

Zusammenfassung: Die Europäische Kommission hat mit Datum 14. September 2016 einen (derzeit stark kontrovers diskutierten) Vorschlag für eine neue Urheberrechtsrichtlinie „On Copyright in the Digital Single Market“ (COM (2016) 593 final) vorgelegt. Dies ist keine Ersetzung der bestehenden Richtlinien, insbesondere nicht der alten InfoSoc-Richtlinie von 2001. Vielmehr wird eine Vielzahl neuer Schrankenregelungen vorgeschlagen (z.B. für grenzüberschreitende Ausbildung, TDM, Gedächtnisorganisationen, Bibliotheken, Leistungsschutzrecht für Presseverleger) die jetzt weitgehend obligatorisch für eine Umsetzung in das jeweils nationale Recht der Mitgliedsländer sein sollen. In diesem Beitrag steht die Frage im Zentrum, inwieweit der jetzige Vorschlag allgemein zu einer Verbesserung der Nutzungssituation für Wissen und Information in Bildung und Wissenschaft führen kann.

Deskriptoren: Europa, Europäische Union, Urheberrecht, Wissenschaft und Technik, Bildungswesen, Gesetz, Vervielfältigung, Vergütung, Nutzung

\section{The proposal of the Commission of the European Commu-} nities for a new Copyright Directive

Abstract: As of September 14th 2016, the European Commission published a proposal for a new Copyright Directive „On Copyright in the Digital Single Market“ (COM(2016) 593 final) which is momentarily rather controversially being discussed. This Directive is not supposed to replace the existing Directives. In particular, the old InfoSoc-Directive from 2001 still continues to apply. The Commission proposes a set of new exceptions and limitations (for instance, for education, TDM, memory organizations, libraries, ancillary copyright for press publishers) which are

*Kontaktperson: Prof.-em. Dr. Rainer Kuhlen, Bogotastraße 4, 14163 Berlin, E-Mail: rainer.kuhlen@uni-konstanz.de, http://orcid.org/0000-0002-4497-6422 mandatory for being transferred into respective national copyright laws of the EU member states. This article mainly deals with how this proposal can contribute to a more education- and research-friendly copyright.

Keywords: European Union, European Commission, copyright, science, technology, memory organizations, libraries, text and data mining (TDM), cross-border education, ancillary copyright for press publishers, reproduction, making publicly available, remuneration, licensing, use, knowledge and information

\section{La proposition de la Commission européenne pour une} nouvelle directive sur le droit d'auteur. La vue de l'exploitation économique continue de primer sur les formes d'échanges ouverts dans l'éducation et les sciences

Résumé: La Commission européenne a présenté le 14 septembre 2016 une proposition pour une nouvelle directive dans le domaine du droit d'auteur Le droit d'auteur dans le marché unique numérique (COM (2016) 593 final). La question centrale de cet article est de savoir dans quelle mesure la proposition actuelle peut conduire à une amélioration de la situation de l'utilisation des connaissances et de l'information dans l'éducation et les sciences. D'autres aspects de la proposition de l'UE sont également brièvement analysés.

Descripteurs: Europe, Union européenne, droit d'auteur, science et technologie, éducation, loi, reproduction, rémunération, utilisation

\section{Vorbemerkung}

Das Urheberrecht liegt nach wie vor in der Zuständigkeit der jeweiligen nationalen Gesetzgebung der einzelnen EULänder, obgleich es immer wieder Bestrebungen gibt, ein einheitliches EU-Recht dafür zu schaffen. Bislang nimmt die EU ihre Regulierungskompetenz für das Urheberrecht 
über Richtlinien wahr, die i.d.R. innerhalb von zwei Jahren in nationales Recht umzusetzen sind. ${ }^{1}$

In den Fußnoten 7 bis 13 des EU-Vorschlags sind die seit 1996 auf das Urheberrecht bezogenen Richtlinien der EU dokumentiert. Der jetzige Vorschlag ist laut der Kommission kompatibel mit diesen weiterhin geltenden Richtlinien, vor allem mit der zentralen sogenannten InfoSoc Richtlinie von $2001^{2}$, ersetzt diese also nicht. Alle diese Richtlinien und der jetzige Entwurf sollen die Funktionsfähigkeit des internen EU-Marktes unterstützen, nicht zuletzt dadurch, dass ein hohes Schutzniveau für die Rechtsinhaber eingerichtet wird: „ensure a high level of protection for right holders and facilitate the clearance of rights“ (p. 4). Mit „Rechtsinhaber“ sind, so legt es zumindest das europäische Urheberrecht nahe, die Autorinnen und Autoren gemeint, die den Schutz ihrer Werke nicht nur über die Persönlichkeitsrechte, sondern auch exklusiv den Schutz ihrer Verwertungsrechte genießen. Dem ist auch so. Aber faktisch reklamieren in den meisten Fällen die Verleger diesen Schutz, weil sie bis in die jüngste Vergangenheit, aber oft genug auch heute noch per Vertrag von den Autorinnen und Autoren deren Verwertungsrechte als Nutzungsrechte ebenfalls mit exklusivem Anspruch übernommen haben.

Verlage (davon sind die auf den Bildungs- und Wissenschaftsmärkten nur ein kleiner, aber nicht zu vernachlässigender Teil) sind Teil des Publikations-/Medienmarktes. Der Bezug auf den internen Markt dient entsprechend auch der EU-Kommission als die entscheidende Legitimation für die Urheberrechts-/Copyright-Regulierung in der EU. Der Eindruck ist allerdings kaum von der Hand zu weisen, dass die Regulierung des Urheberrechts-/Copyrights sehr stark durch die Sicht auf die Publikumsmärkte geprägt wird.

Wie auch immer - die bisherigen Regulierungen haben allerdings kaum zu dem von der EU-Kommission als zentral angesehenen Marktziel der Harmonisierung/ Vereinheitlichung des Urheberrechts in den EU-Ländern geführt. ${ }^{3}$ Vieles, vor allem in der InfoSoc Richtlinie, ist für

1 Als dieser Beitrag geschrieben wurde, lag noch keine autorisierte deutsche Fassung vor. Es wird daher hier in erster Linie aus der (allgemein auch als prioritär angesehenen) englischen Fassung zitiert: http://ec.europa.eu/newsroom/dae/document.cfm?doc_id=17200.

Alle Sprachversionen des Vorschlags jetzt unter: http://eur-lex.euro pa.eu/legal-content/EN/TXT/?uri=CELEX:52016PC0593.

2 Directive 2001/29/EC of the European Parliament and of the Council of 22 May 2001 on the harmonisation of certain aspects of copyright and related rights in the information society (OJ L 167, 22.6.2001, p. 10-19).

3 Vgl. Mireille van Eechoud: P. Bernt Hugenholtz; Stef van Gompel; Lucie Guibault; Natali Helberger: Harmonizing European Copyright die Umsetzung in das jeweilige nationale Urheberrecht optional, weniges obligatorisch - mit der Folge, dass das Urheberrecht in den EU-Ländern weiterhin doch arg flickenteppichähnlich ist.

Der jetzige Vorschlag der EU-Kommission bevorzugt tendenziell obligatorische Lösungen (verwendet verschiedentlich aber auch „may provide“), allerdings ohne damit die bestehenden optionalen Regulierungen durchgängig in verbindliche umzuwandeln. Das gilt in unserem Zusammenhang vor allem für die Ausnahme/Begrenzung ${ }^{4}$ in Art. 5, 3, a welche die (optionale) Vorgabe für nationale Schranken für Bildung und Wissenschaft ist - in Deutschland § 52a UrhG (vgl. Abschnitt 2.3):

\begin{abstract}
„a) für die Nutzung ausschließlich zur Veranschaulichung im Unterricht oder für Zwecke der wissenschaftlichen Forschung, sofern - außer in Fällen, in denen sich dies als unmöglich erweist - die Quelle, einschließlich des Namens des Urhebers, wann immer dies möglich ist, angegeben wird und soweit dies zur Verfolgung nicht kommerzieller Zwecke gerechtfertigt ist“.
\end{abstract}

Seit etwa zehn Jahren hat die Diskussion über ein neues Urheberrecht in der EU begonnen, wie üblich anfänglich mit einem Grünbuch der Kommission ${ }^{5}$. Es hat eine Weile gedauert, bis sich die Kommission zu einer öffentlichen Befragung (consultation) zum Urheberrecht entschieden hatte. Der ausführliche Auswertungsbericht wurde 2014 öffentlich zugänglich gemacht. ${ }^{6}$ Große Erwartungen erweckte der Beschluss des Europäischen Parlaments von

Law. The Challenges of Better Lawmaking. Information Law Series. Vol. 19. Kluwer Law International 2009 - http://www.ivir.nl/publica ties/download/967.pdf.

4 Im Englischen werden die Einschränkungen der exklusiven Rechte der Rechtsinhaber „exceptions“ oder „limitations“ genannt (oft auch beides zusammen). Ich bevorzuge die Verwendung von „limitations“. Einschränkungen sollten nicht als Ausnahmen verstanden werden; vielmehr sind sie im Urheberrecht ausdrücklich als im öffentlichen Interesse stehende Begrenzungen (limitations) der Rechte der Rechtsinhaber vorgesehen. Sie sind systematischer Bestandteil des Urheberrechts. In Deutschland wird im Allgemeinen von Schranken bzw. Schrankenbestimmungen gesprochen. Auch das ist keine glückliche Benennung. Schranken sollen gerade die Nutzung im öffentlichen Interesse teilweise frei, wenn auch nicht unbedingt vergütungsfrei machen. Sie sollten nicht als ärgerliche Einschränkungen der exklusiven Verwertungs-/Nutzungsrechten verstanden werden. Erfreulicherweise verwendet in Deutschland das BMJV in seinem aktuellen Referentenentwurf für ein Wissenschaftsurheberrecht anstelle von „Schranken“ die Formulierung „Gesetzlich erlaubte Nutzungen für Unterricht, Wissenschaft und Institutionen“ - https://www.bmjv.de/SharedDocs/Gesetz gebungsverfahren/Dokumente/RefE_UrhWissG.pdf.

5 http://ec.europa.eu/internal_market/copyright/docs/copyright-inf so/greenpaper_en.pdf.

6 Report on the responses to the Public Consultation on the Review of the EU Copyright Rules. July 2014 - http://ec.europa.eu/internal_ 
Mitte 2015. ${ }^{7}$ Dieser Parlaments-Beschluss beruhte auf einem fulminanten, schlussendlich dann aber in vielen Details vom Parlament zurückgenommenen Report von Julia Reda (Mitglied des EU-Parlaments für die Piratenpartei). ${ }^{8}$

Nach all diesen Vorgaben hatte die Fachöffentlichkeit gehofft oder sogar erwartet, dass durch die jetzt angestoßene Urheberrechtsreform in der EU die InfoSoc-Richtlinie von 2001 gänzlich ersetzt würde. 2001 ist im Zeitalter des Internet gefühlt so weit entfernt wie die Gegenwart vom Mittelalter, zumal die Diskussionen über diese sogenannte InfoSoc-Richtlinie weit in die 1990er Jahre zurück reichen. Nicht nur haben sich die politischen, technologischen und ökonomischen Rahmenbedingungen, sondern auch die moralischen, informationsethisch begründeten Verhaltensformen ${ }^{9}$ für den Umgang mit Wissen und Information radikal verändert, so dass ein wirklicher Neuansatz nötig gewesen wäre.

Als unter der Verantwortlichkeit des damals für die Digitalwirtschaft zuständigen EU-Kommissars Günther Oettinger der Vorschlag im Herbst 2016 öffentlich wurde, machte schon dessen Titel deutlich, dass das Urheberrecht weiter in den Kontext von Digital Single Market gestellt werden sollte. Offensichtlich wollte die EU-Kommission an dem alten Paradigma festhalten, dass das Urheberrecht in erster Linie dazu dienen soll, den europäischen Binnenmarkt für Wissen und Information nicht nur funktionsfähig zu halten, sondern ihn auch in seinem weiteren Ausbau und mit starken Schutzbestimmungen zu befördern. Das wird vor allem an den Art. 11 bis 13 deutlich, durch die nach Einschätzung vieler Wissen-

market/consultations/2013/copyright-rules/docs/contributions/con sultation-report_en.pdf.

7 http://www.europarl.europa.eu/sides/getDoc.do?type=TA\&refere nce=P8-TA-2015-0273\&language $=E N$.

8 European Parliament resolution of 9 July 2015 on the implementation of Directive 2001/29/EC of the European Parliament and of the Council of 22 May 2001 on the harmonisation of certain aspects of copyright and related rights in the information society"; ursprüngliche Version: https://juliareda.eu/copyright-evaluation-report-explained/; die endgültige unter: https://juliareda.eu/copyright-evaluation-re port/full/; vgl. dazu Rainer Kuhlen: Kopernikanische Wende in der EU-Urheberrechtsdebatte? Die Politik in Deutschland sollte das nutzen. netzpolitik.org 21.1.2015 - https://netzpolitik.org/2015/kopernika nische-wende-in-der-eu-urheberrechtsdebatte-die-politik-in-deutsch land-sollte-das-nutzen/.

9 Vgl. Rainer Kuhlen: Interdependenzen zwischen Informationsethik und politischem Handeln - am Beispiel einer kontroversen Urheberrechtsregulierung (Zweitverwertungsrecht). In: Zeitschrift für Politikwissenschaft (ZPol), Sonderband Ethik und Politikmanagement, 2014, 193-221, Preprint: http://www.kuhlen.name/MATERIALIEN/Pu blikationen2014/interdependenzen-informationsethik-urheberrechtPDF.pdf. schaftler allerdings eher Innovationen behindert als befördert würden. ${ }^{10}$

Diese Funktionalisierung des Urheberrechts ist, wie zu Anfang erwähnt, der EU-Urheberrechtsregulierung immanent. Sie müsste auch nicht unbedingt kritisiert werden wenn denn unter „Märkten“ nicht nur die kommerziellen, auf den Schutz proprietärer Eigentumsrechte pochenden Verwertungsformen verstanden, sondern auch die das Internet gleichermaßen bestimmenden offenen, auf freien Zugang zu und freier Nutzung von Wissen und Information setzenden Austauschformen eingeschlossen würden. ${ }^{11}$

Leider hat sich die EU-Kommission nur sehr bedingt auf diesen Paradigmenwechsel - Zusammenspiel der kommerziellen und der offenen Informationsmärkte - eingelassen. Dies wird besonders bei den auf Bildung und Wissenschaft bezogenen Vorschlägen deutlich. Sie sind zwar teilweise als Fortschritt anzuerkennen. Aber selbst diese bleiben auf halbem Wege stecken, so dass sie in dieser Form kaum wirkliche informationelle Mehrwerteffekte für Bildung und Wissenschaft bewirken werden.

Schon gar nicht ist eine umfassende Neuregelung der Nutzungsbedingungen in Bildung und Wissenschaft vorgelegt worden. Die europaweite Diskussion um ein allgemeines umfassendes Nutzungsprivileg über eine Allgemeine Bildungs- und Wissenschaftsschranke (vgl. Abschnitt 2.3) wurde von der EU-Kommission nicht aufgegriffen.

\section{Zusammenfassung}

Der EU-Kommission ist es, trotz einiger positiv einzuschätzenden, aber doch wieder stark eingeschränkten Weiter-

10 Art 11 schlägt ein neues Leistungsschutzrecht für Presseverleger vor (vgl. Abschnitt 2.4); durch Art. 12 soll der Verlegeranteil an Vergütung für schrankenbezogene Nutzungen gesichert werden (vgl. Abschnitt 2.5) und durch Art. 13 soll durch eine Änderung der Commerce Directive (2000/31/EC) die Verantwortlichkeit bzw. die Haftung für nutzergenerierte Inhalte zwischen den Rechtsinhabern und Service Provider geteilt werden. Vor allem die Artikel 11 und 13 sollten nach Einschätzung vieler Wissenschaftler besser ganz gestrichen werden, vor allem weil sie sich als innovationsfeindlich erweisen würden. (vgl. z.B.http://copyright4creativity.eu/2017/02/24/imco-opinion-oncopyright-in-the-digital-single-market-things-are-looking-better-butthe-devil-is-still-in-the-details/oder http://ancillarycopyright.eu/new s/2017-02-23/leading-european-copyright-scholars-fundamentally-cri ticise-ancillary-copyright).

11 Rainer Kuhlen: Informationsmarkt. Chancen und Risiken der Kommerzialisierung von Wissen. Schriften zur Informationswissenschaft Vol. 15. Universitätsverlag Konstanz (UVK), Konstanz 1995. Kap. 1 Von den zwei Informationsmärkten der Ökonomie und der Wissenschaft, S. $3 \mathrm{ff}$. 
entwicklungen insgesamt nicht gelungen, einen der gegenwärtigen Praxis der Produktion und Nutzung von Wissen und Information gerecht werdenden Vorschlag oder gar einen in die Zukunft weisenden Paradigmenwechsel für die Urheberrechtsregulierung vorzulegen. Urheberrecht wird in erster Linie als Vehikel zur Beförderung der digitalen Wirtschaft angesehen.

Auch die EU-Kommission, ebenso das EU-Parlament und der EU-Rat, sollten die offenen, auf freien Zugang zu und freier Nutzung von Wissen und Information setzenden Austauschformen berücksichtigen - nicht nur, aber vor allem auch in Bildung und Wissenschaft. Das Interesse von Gesellschaft und Wirtschaft daran sollte höher gewichtet werden als der Schutz bestehender Geschäftsmodelle und Finanzierungsformen der Publikationswirtschaft.

Im Einzelnen:

1) Der in Art. 6 des Vorschlags - mit Referenz auf InfoSoc 2001, Art. 5, 5 und Art. 6, 4, para 3 - weiter bestehende Schutz technischer Schutzmaßnahmen (Digital Rights Management - DRM) und damit die Ermunterung zum Einsatz dieser Maßnahmen ist in Bildung und Wissenschaft nicht angemessen. Die durch Schrankenbestimmungen legitimierten Nutzungsformen für Bildung und Wissenschaft sollten nicht von freiwilligen Maßnahmen der Rechtsinhaber abhängen, sondern sollten alleine durch das Recht direkt garantiert werden. (ausführlicher Abschnitt 1.1)

2) Die Einführung einer Ausnahme/Schrankenbestimmung für Text and Data Mining (TDM) im EU-Vorschlag, Art. 3 wird aus Gründen der Rechtssicherheit begrüßt. Fraglich ist allerdings, ob eine solche Schranke an sich überhaupt nötig ist. Aus dem Recht zu lesen (read) (r2r) sollte auch das Recht zum „mining“ (r2m) folgen. Die Beschränkung der TDM-Nutzung auf „scientific research“ und damit die Ausklammerung von kommerziellem TDM wird für nicht gerechtfertigt und nicht für zielführend gehalten. Genutzte TDMKorpora sollten nicht nur archiviert (i. d. R. von Bibliotheken), sondern auch (nur) für eine anfällige Qualitätsüberprüfung verfügbar gemacht werden. (ausführlicher vgl. Abschnitt 1.2)

3) Die Regelungen für ,digital and cross-border teaching activities“ im EU-Vorschlag, Art. 4 für Ausbildungseinrichtungen auf allen Ebenen werden im Prinzip begrüßt, insbesondere auch die Nutzung für die die Lehre begleitenden Aktivitäten und Prüfungssituationen. Letzteres sollte aber in Art. 4 explizit gesichert werden. Ein Fortschritt ist es, dass jetzt der externe, gesicherte und autorisierte Zugriff auf die Materialien der Bibliothek nicht ausgeschlossen ist. Die Einschrän- kung, dass der externe Zugriff nur aus dem Land, in dem die Ausbildungseinrichtung angesiedelt ist, erlaubt sei, ist allerdings mit der Idee des ,cross-borderteaching" nicht vereinbar. In Art. 4, wie auch in anderen Artikeln, ist eine zu kritisierende Tendenz erkennbar, die Priorität einer rechtlich verbindlichen Schrankenregelung zugunsten von Lizenzvereinbarungen auszuhebeln. Die durch Art. 4, 4 gegebene Möglichkeit sollte bei nationalen Umsetzungen genutzt werden, so dass für Ausbildungszwecke eine vergütungsfreie Nutzung vorgesehen werden könnte. (ausführlicher vgl. Abschnitt 1.3 und 2.6)

4) Die EU sollte aus der Begünstigung von behinderten Personen durch Art. 4 die Konsequenz ziehen, die (bislang nur optionale) Schrankenregelung in InfoSoc 2001 Art. 5, 3, b auch als obligatorisch für Wissenschaft zu erklären.

5) Das durch den EU-Vorschlag Art. 5 eingeführte (an sich selbstverständliche) Recht auf digitale Vervielfältigungen von im Bestand der Bibliotheken vorhandenen Werken zur Bewahrung des kulturellen Erbes wird aus Gründen der Rechtssicherheit begrüßt. Der Vorschlag versäumt es aber festzulegen, in welcher Form diese neuen Vervielfältigungen auch genutzt werden dürfen. (ausführlicher vgl. Abschnitt 1.4)

6) Für die Nutzung vergriffener Werke soll (wie schon in Deutschland geschehen) im EU-Vorschlag Art. 7 eine die Regelung für verwaiste Werke ergänzende Schrankenregelung zur Nutzung von vergriffenen Werken eingeführt werden. Dies ist im Prinzip zu begrüßen. Jedoch ist dieser Artikel, vor allem bezüglich der Lizenzierungsbedingungen, so komplex und unverständlich angelegt, dass er kaum Klarheit für die Nutzung vergriffener Werke schaffen kann. (ausführlicher vgl. Abschnitt 1.5)

7) In Kapitel 2 werden weitere Kritikpunkte aufgeführt:

- Es ist nicht klar, welche bisherigen Schrankenbedingungen weiter gelten sollen bzw. welche nun optional bleiben und welche obligatorisch sein sollen. (vgl. Abschnitt 2.1)

- Notwendige Regelungen für Ausleihe und Versand digitaler Objekte (e-Books) durch Bibliotheken bleiben ausgeklammert. (vgl. Abschnitt 2.1)

- Der Ankündigung zur Senkung von Transaktionskosten für die Wissenschaft folgen keine konkreten Maßnahmen. (vgl. Abschnitt 2.2)

- Es liegt kein Vorschlag vor, wie die zu allgemein gehaltene Schrankenregelung aus InfoSoc 5, 3, a durch eine Allgemeine Bildungs- und Wissenschaftsschranke (ABWS) zu ersetzen ist. (vgl. Abschnitt 2.3) 
- Die Einführung eines Leistungsschutzrechts für Presseverleger wird nicht zuletzt als eine Einschränkung von Link- und Informationsfreiheit für Bildung und Wissenschaft eingeschätzt. (vgl. Abschnitt 2.4)

- Es ist zu bezweifeln, ob, wie jetzt vorgesehen, die Beteiligung der Verlage an den Ausschüttungen der Verwertungsgesellschaften durch das Urheberrecht geregelt werden soll/darf. (vgl. Abschnitt 2.5)

- Angesichts der besonderen Situation von Bildung und Wissenschaft sollte das Problem der Vergütung differenziert gelöst werden. Wenn Vergütung überhaupt vorgesehen sein soll, sollte dies nur über pauschale Abrechnungsformen geschehen. (vgl. Abschnitt 2.6)

\section{Zu den Bildung und Wissenschaft direkt betreffenden Vorschlägen der EU-Kommission}

In Erwägungsgrund 5 (im Folgenden als EG abgekürzt) ${ }^{12}$ des Vorschlags bestätigt die Kommission, dass die bislang vor allem durch die InfoSoc-Richtlinie von 2001 geltenden Schrankenbestimmungen nicht mehr zeitgemäß sind und dass sie entsprechend neu bewertet werden müssen. ${ }^{13}$ Die konkreten vorgeschlagenen Regulierungsvorschriften sollten an dieser Aussage gemessen werden. Das soll im Folgenden unternommen werden.

\subsection{Umgang mit technischen Schutzmaßnahmen}

Maßnahmen: Der durch InfoSoc 2001 im dritten Absatz von Art. 6, 4 eingeführte Schutz technologischer Schutzmaßnahmen (DRM) soll nach Einsicht der EU-Kommission

12 Erwägungsgründe (EG) sind Erläuterungen zu den einzelnen Artikeln von Gesetzen oder Richtlinien. Sie sind nicht Teil des rechtlich verbindlichen Textes der Artikel. Sie werden aber i.d.R. bei Streitfällen vor Gerichten verwendet, um die Absicht des Gesetzgebers zu verstehen und dieser i.d.R. auch zu folgen.

13 „In the fields of research, education and preservation of cultural heritage, digital technologies permit new types of uses that are not clearly covered by the current Union rules on exceptions and limitations. ... Therefore, the existing exceptions and limitations in Union law that are relevant for scientific research, teaching and preservation of cultural heritage should be reassessed in the light of those new uses." (Vorschlag der Kommission, Erwägungsgrund 5). weiter bestehen bleiben. Die Rechtsinhaber sollen dafür sorgen, dass die durch die Schrankenbestimmungen des Rechts legitimierten Nutzungsformen weiter möglich bleiben. Allerdings bleibt es ihnen über ,freiwillige Maßnahmen“ überlassen, welches Format und welche Modalitäten sie dafür als geeignet ansehen (vgl. Erwägungsgrund 7). Nur wenn diese freiwilligen Maßnahmen nicht bereitgestellt werden, sollen die Mitgliedsstaaten Maßnahmen vorsehen, um die im Recht vorgesehenen Nutzungen zu ermöglichen. Wie das geschehen soll, wird trotz des Verweises auf Art. 6, 4 von InfoSoc 2001 nicht gesagt.

\section{Kritik:}

- Der weiter bestehende Schutz technischer Schutzmaßnahmen (DRM) und damit die Ermunterung zum Einsatz dieser Maßnahmen sind in Bildung und Wissenschaft nicht angemessen.

- Die durch Schrankenbestimmungen legitimierten Nutzungsformen für Bildung und Wissenschaft sollten nicht von freiwilligen Maßnahmen der Rechtsinhaber abhängen, sondern sollten alleine durch das Recht direkt garantiert werden.

- Vor allem mit Rücksicht auf die Ausbildung, bei der z.B. für Lehrveranstaltungen üblicherweise eine Vielzahl von Materialien verschiedener Rechtsinhaber genutzt wird, ist es nicht zumutbar, dass mit jedem Anbieter Verhandlungen über die Beseitigung technischer Maßnahmen geführt werden.

- Der Ausweg, vor Gericht darüber entscheiden zu lassen, ob die Aufhebung einer DRM-Maßnahme gerechtfertigt ist, ist aus Zeitgründen kaum praktikabel; die jeweilige Lehrveranstaltung dürfte dann längst vorbei sein.

- Auch wird die Bibliotheksarbeit dadurch stark behindert, wenn selbst einfache Vorgänge wie Vervielfältigungen zur Sicherung des kulturellen Erbes bei DRMBeschränkungen nicht durchgeführt werden können.

DRM ist ohne Frage ein Relikt aus der Zeit, zu der die Furcht vor Piraterie dominanter war als die Energie, neue Produkte und Dienstleistungen auf den Markt zu bringen. Die Musik- und Videoindustrien haben mit ihrem (aber durchaus nicht vollständigen) Verzicht auf DRM diese Argumentation weitgehend aufgegeben und sich so neue Märkte z.B. über niedrigpreisiges Streaming mit hohen Renditen erschlossen. Auch der Wissenschaftsmarkt sollte eher den innovativen als den ausschließenden Weg gehen. Die Rechtsetzung sollte den restriktiven DRM-Gedanken aufgeben bzw. im Zweifelsfall der Schrankenregelung Vorrang einräumen. 


\subsection{Neue Schrankenbestimmung für Text and Data Mining (TDM)}

Maßnahme: Die EU-Kommission sieht hier Handlungsbedarf. Es sei nicht auszuschließen, dass, angesichts der wachsenden Bedeutung von TDM, Rechtsinhaber bei Lizenzen für große Text- und Datensammlungen TDM ausschließen bzw. dafür neue Vereinbarungen aushandeln wollen.

Für die EU war es offenbar nicht eindeutig geklärt, ob und inwieweit den TDM-Aktivitäten von Forschungseinrichtungen Urheberrechts- bzw. Datenbankschutz-Bedenken entgegenstehen. Vermutlich reagiert die EU mit ihrem Vorschlag auch auf das schon 2014 erfolgte Vorpreschen des UK in Sachen TDM. ${ }^{14}$ Die EU-Kommission möchte mit Art. 3 des Vorschlags rechtliche Unsicherheit beseitigen und eine für alle Mitgliedsstaaten verbindliche Schrankenregelung einführen.

\begin{abstract}
„Member States shall provide for an exception to the rights provide for in Article 2 of Directive 2001/29/EC, Articles 5(a) and 7(1) of Directive 96/9/EC and Article 11(1) of this Directive for reproductions and extractions made by research organisations in order to carry out text and data mining of works or other subjectmatter to which they have lawful access for the purposes of scientific research.“(EU-Vorschlag, Art, 3, 1)
\end{abstract}

Die bisherige verbindliche Schrankenbestimmung für vorübergehende Vervielfältigungen (temporary acts) bleibt erhalten. (EG 10) Allerdings fallen TDM-Anwendungen in der Wissenschaft wohl kaum unter diese „temporary acts“. Die neue Schranke ergänzt durch Art. 3, 1 die Rechte, die z. B. durch InfoSoc Art. 5, a gegeben sind. Vertragliche Bestimmungen, die der neuen Schranke entgegenstehen, können nicht erzwungen werden (unenforceable). (Art. 3, 2)

Von der neuen Schranke sollen auch Forschungsorganisationen Nutzen haben, die in ,public-private partnerships"-Vorhaben involviert sind. (EG 10) Organisationen, die TDM unter rein kommerziellen Aspekten betreiben, werden aber nicht als Forschungsorganisationen angesehen und bleiben von der Schranke ausgeschlossen.

Ganz frei soll eine Nutzung nach dieser Schranke allerdings nicht sein: Den Rechtsinhabern wird das Recht eingeräumt, Maßnahmen zu ergreifen, wenn durch den Umfang des zu nutzenden Materials die Sicherheit und Integrität des Systems bzw. der Datenbank gefährdet ist. (Art. 3, 3) Rechtsinhaber und Forschungsorganisationen

14 Vgl. https://www.gov.uk/government/uploads/system/uploads/ attachment_data/file/375954/Research.pdf. sollen sich bezüglich der Anwendung solcher Maßnahmen einvernehmlich verständigen. (Art. 3, 4)

Für die TDM-Nutzung soll (bemerkenswert!) keine Entschädigung (Vergütung) erfolgen, weil der Schaden für die Rechtsinhaber durch TDM-Nutzung minimal sei. (EG 13) Zu beachten ist auch, dass die Schrankenerlaubnis für TDM keineswegs einen Freibrief für illegale Nutzungshandlungen (Piraterie) gibt. Wie bei anderen Schranken handelt es sich auch bei der für TDM immer um Nutzungen von bereits (i.d.R. von Bibliotheken) auf legalem Wege (Kauf oder Lizenz) erworbenen Materialien.

Kritik: Neben dem Schrankenvorschlag für ein Leistungsschutzrecht für Presseverleger in Art. 11 (vgl. Abschnitt 2.4) hat der TDM-Vorschlag in Art. 3 besonders vielfältige Kritik provoziert:

- Es ist umstritten, ob es eine Notwendigkeit für eine Schrankenbestimmung für TDM gibt, wenn für TDM ein legaler Zugriff auf die zu analysierenden Materialien schon besteht. Wenn man diese berechtigterweise lesen darf, warum soll man sie nicht auch analysieren dürfen - so die Argumentation. Die Allianz der deutschen Wissenschaftsorganisationen vertritt z.B. den Standpunkt, dass es sich bei TDM um eine urheberrechtlich nicht relevante Handlung handelt. Zudem ist durchaus strittig, ob das Urheberrecht durch TDM-Aktivitäten überhaupt betroffen ist. Bekanntlich sind Daten, Fakten, Ideen nicht urheberrechtlich geschützt. ${ }^{15}$ Wie dem auch sei - aus der Sicht von Bildung und Wissenschaft ist die Einführung dieser Regelung sinnvoll, wenn sie der Rechtssicherheit dient.

- Es wird allerdings für einen Fehler gehalten, das neue TDM-Recht auf „research organisations ... for the purposes of scientific research" $\mathrm{zu}$ beschränken (gemeint ist Forschung in nicht-kommerzieller Absicht). Diese Einschränkung würde einen erheblichen Wettbewerbsnachteil der europäischen Wirtschaft gegenüber Ländern wie USA oder Japan nach sich ziehen (in den USA ist TDM durch die Fair-Use-Doktrin weitgehend gedeckt). Auch würde sie die erforderliche und erwünschte Zusammenarbeit der öffentlich finanzier-

15 Vgl. EDiMA, the European association representing European and global online platforms and other innovative tech companies operating in the EU: „Maintaining that facts and ideas be protected by copyright goes against international legal standards as copyright protection does not extend to factual information about a work. Any copying in the context of TDM is incidental“ and does not result in any unreasonably harm to the legitimate interests of the copyright holder - http://edima-eu.org/pdfs/latest_news/EDIMA\%20copyright \%20leave\%20behind\%20final\%20wout\%20ES\%20reference.pdf. 
ten bzw. im öffentlichen Interesse liegenden Forschung mit industrieller Forschung zwar nicht ganz ausschließen (erlaubt scheint sie zu sein, wenn die industrielle Forschung nicht direkt kommerziellen Zwecken dient ${ }^{16}$ ), aber vermutlich doch stark behindern. Eine solche Behinderung, vom BDI als ,wirtschafts- und realitätsferne Verengung“ abgelehnt ${ }^{17}$, steht im Widerspruch zu dem allgemeinen Ziel der EU, den digitalen Binnenmarkt zu befördern.

- Die vorgesehene Möglichkeit, dass die Rechtsinhaber Maßnahmen ergreifen können, wenn sie durch starke, z.B. große Datenmengen umfassende TDM-Nutzung die „Sicherheit und Integrität“ ihrer Systeme beeinträchtigt sehen, droht die Schranke auszuhebeln, weil die meisten TDM-Anwendungen auf „big data“, also auf umfassender Datennutzung, beruhen. Die Aufforderung, dass sich Rechtsinhaber und TDM-Anwender über solche Maßnahmen verständigen, würde Forschungsorganisationen in langwierige Verhandlungen verstricken, welche zeitnahe Anwendungen behindern. Auch TDM-Forschung sollte so frei wie irgend möglich sein. Zudem ist es mehr als fraglich, ob Sicherheit und Integrität über das Urheberrecht, wie hier vorgesehen, geregelt werden soll. Schon gar nicht sollte dieses Zugeständnis an die Rechtsinhaber dazu führen, dass der Umfang von Mining oder eine mögliche Verwertung eingeschränkt wird. Ob Rechtsinhaber einen Anspruch auf Teilnahme an möglichen Einnahmen aus den TDM-Analysen haben sollen, müsste vermutlich noch geklärt werden.

- Als Bedingung für Wissenschaftlichkeit muss Vorsorge dafür getroffen werden, dass die in der Wissenschaft durch TDM erzielten Forschungsergebnisse nachprüfbar sind. Es ist also keineswegs mit einem

16 Forschungsorganisationen werden im EU-Vorschlag Art. 2, 1 wie folgt definiert: ,'research organisation' means a university, a research institute or any other organisation the primary goal of which is to conduct scientific research or to conduct scientific research and provide educational services:

(a) on a non-for-profit basis or by reinvesting all the profits in its scientific research; or

(b) pursuant to a public interest mission recognised by a Member State;

in such a way that the access to the results generated by the scientific research cannot be enjoyed on a preferential basis by an undertaking exercising a decisive influence upon such organisation".

17 BDI (Spitzenorganisation der deutschen Industrie und der industrienahen Dienstleister) - https://www.bmjv.de/SharedDocs/Gesetz gebungsverfahren/Stellungnahmen/2016/Downloads/11112016_Stell ungnahme_BDI_EU-Urheberrechtsreform.pdf?_blob=publicationFil $\mathrm{e} \& \mathrm{v}=2$. temporären Recht auf Vervielfältigung getan. Bibliotheken sollte die für TDM erstellten Korpora dauerhaft speichern und für neue Forschungsfragen bzw. zumindest zur Überprüfung der publizierten Ergebnisse wieder bereitstellen dürfen. Ob dafür die vom BDI vorgeschlagene „Offenlegung sogenannter „de minimis“Teile der analysierten Texte bzw. Daten in Form von „Snippets“ in der Praxis“ (vgl. FN 17) ausreichend sind, kann bezweifelt werden.

Insgesamt scheint die Diskussion um eine TDM-Schranke noch nicht umfassend genug geführt zu sein. Z. B. besteht die Gefahr, dass durch eine neue Schranke implizit ein neues Schutzrecht eingeführt würde.

\subsection{Schrankenregelung zugunsten "cross-border teaching activities“}

Art. 4 schlägt Regelungen für die Nutzung von Werken und anderen Materialien ,in digital and cross-border teaching activities" vor.

Maßnahme: Der Vorschlag der EU-Kommission versucht Klarheit in die bisherigen Vorgaben aus InfoSoc 2001 (hier Art. 5, 3, 1) und der Datenbankrichtlinie (hier Art. 6, 2, b und 9, b) zu bringen - allerdings nur mit Blick auf Ausbildung und insbesondere für ,distance and cross-border teaching“. (EG 14) Dazu sei eine neue, für alle Länder verbindliche Schrankenregelung erforderlich.

Begünstigt werden sollen Ausbildungseinrichtungen auf allen Ebenen (primary, secondary, vocational and higher education). (EG 15) Nicht der institutionelle Status sei entscheidend, sondern nur der nicht-kommerzielle Zweck der Ausbildung. Umfassend gilt allerdings nur das Vervielfältigungsrecht. Dazu stellt Art. 17, 2, a klar, dass die Formulierung aus InfoSoc 2001, Art. 5, 2 so geändert werden soll, dass Vervielfältigungsrechte auch für Ausbildungseinrichtungen (neben, Bibliotheken, Archiven und Museen) eingeräumt werden können. Das sollte dann auch bei den anfälligen nationalen Urheberrechtsreformen aufgegriffen werden.

Die digitale Nutzung ist nur für Teile oder Auszüge von Werken vorgesehen. So muss wohl EG 16 verstanden werden. Aber sie ist erlaubt nicht nur für das Lehren, sondern auch für „related learning activities“, insbesondere auch für Prüfungssituationen. Die Nutzung beschränkt sich nicht länger auf die Unterrichtsumgebung (,in the classroom"), sondern erlaubt auch die externe authentifizierte Nutzung über ein sicheres Netzwerk der Ausbildungseinrichtung. (EG 16) 
Nicht zuletzt sollen auch Personen mit Behinderungen durch Art. 4 begünstigt werden: ,The exception or limitation should be understood as covering the specific accessibility needs of persons with a disability in the context of illustration for teaching.“ (EG 16)

Die Schranke räumt den Mitgliedsländern die Möglichkeit ein, kommerziellen, auf Ausbildung bezogenen Lizenzangeboten Priorität gegenüber der Schrankenregelung zu geben, falls diese die in der Schranke formulierten Nutzungen ebenfalls erlauben und wenn den Ausbildungseinrichtungen diese Lizenzen bekannt sind (,are aware of"). (EG 17)

Kritik: Der Vorschlag der EU-Kommission Art. 4, 1 verwendet nach wie vor die Formulierung ,for the sole purpose of illustration for teaching“, die in der Vergangenheit zuweilen zu einer sehr engen Interpretation der erlaubten Nutzung geführt hat - so durch die unglückliche Formulierung „Veranschaulichung im Unterricht“ in § 52a UrhG in Deutschland. Die oben angeführten Erwägungsgründe legen zwar nahe, dass eindeutig die auch vom deutschen Bundesgerichtshof (BGH) verfolgte Bedeutung „Veranschaulichung des Unterrichts“, einschließlich der Vorund Nachbereitung des Unterrichts sowie für Prüfungen, gemeint ist. Der Text in Art. 4 sollte dies aber explizit klarstellen.

Es ist ein Fortschritt, dass in Art. 4, 1, a die lästige und unzeitgemäße Beschränkung auf eine Nutzung „on the premises of an educational establishment“ (so noch in $\S 52 \mathrm{~b}$ deutsches UrhG) zwar nicht ganz aufgehoben, aber über einen sicheren, kontrollierten und authentifizierten Netzwerk-Zugriff erweitert wurde: „through a secure electronic network accessible only by the educational establishment's pupils or students and teaching staff“".

Der externe Zugriff wird aber leider dadurch beschränkt, dass gemäß Art. 4, 3 dieser externe Zugriff nur aus dem Land, in dem die Ausbildungseinrichtung angesiedelt ist, erlaubt sein soll. Dies widerspricht dem im Titel von Art. 4 erwähnten „cross-border teaching“ Ansatz. Diese Einschränkung ist gravierend, unnötig und unzeitgemäß.

Die Formulierungen in Art. 4, 2 können dazu führen, die Priorität einer rechtlich verbindlichen Schrankenregelung zugunsten von Lizenzvereinbarungen auszuhebeln. Die Bedingungen, unter denen Lizenzvereinbarungen bzw. -angebote Vorrang haben sollen, sind nicht ausreichend klar definiert.

Die EU sollte aus der Begünstigung von behinderten Personen durch Art. 4 die Konsequenz ziehen, die (bislang nur optionale) Schrankenregelung in Art. 5, 3, b von InfoSoc 2011 jetzt auch als obligatorisch zu erklären. Dies wäre ein großer Fortschritt für alle behinderten Personen, aber natürlich auch für die in Bildung und Wissenschaft Tätigen.

Die EU hat allerdings bislang den Marrakesch-Ver$\operatorname{trag}^{18}$ nicht unterzeichnet, weil verschiedene Mitgliedsländer (Mitglieder im EU-Council) der Ansicht waren, dass die EU keine exklusive Kompetenz für die Unterzeichnung dieses Vertrags habe. Diese Länder stimmen offenbar nicht allen Regelungen des Vertrags zu. Inzwischen hat der EuGH bzw. der Generalanwalt festgestellt, dass die EU sehr wohl die exklusive Kompetenz zur Unterzeichnung habe. ${ }^{19}$ Möglicherweise sollte dann auch die in InfoSoc 2001 Art. 5, 3, b festgelegte „Beschränkung auf den nicht-kommerziellen Gebrauch wegfallen.“

\subsection{Regelung zur Bewahrung des kulturellen Erbes in Art. 5}

Maßnahme: Kulturellen Gedächtnisinstitutionen soll über eine neue Schranke (Vorschlag der EU-Kommission Art. 5) das Recht eingeräumt werden, von Werken aus ihren eigenen Beständen Kopien (Vervielfältigungen) in einem solchen Umfang anzufertigen, die zur Bewahrung dieser Werke nötig sind. Erwägungsgründe 18 bis 21 präzisieren Art. 5. Es wird (zum Glück) nicht versucht, zu definieren, was unter „kulturellem Erbe“ zu verstehen ist. Auch gibt es keine Zeitbeschränkung, ab welchem Zeitpunkt ein Werk Teil des kulturellen Erbes ist.

Kritik: Diese Schranke schafft zwar Klarheit über ein digitales Vervielfältigungsrecht, das an sich selbstverständlich ist, versäumt es aber festzulegen, in welcher Form, diese neuen Vervielfältigungen auch genutzt werden können.

Kultureinrichtungen wie Bibliotheken, Archive oder Museen verstehen sich aber zunehmend nicht mehr als nur Bewahrungseinrichtungen, sondern auch und insbesondere als Nutzungseinrichtungen, welche ihre nun erlaubten digitalen Bewahrungskopien von bisherigen analogen Beständen auch über externen Zugriff, öffentlich zugänglich machen wollen/sollten.

Diese Nutzung sollte für kontrollierbare und authentifizierbare Zwecke von Forschung und Ausbildung oder für private Zwecke möglich sein - zumal dann, wenn die Originale der erstellten Digitalisate aus welchen Gründen auch immer nicht genutzt werden können/sollen. Diese

18 http://www.wipo.int/wipolex/en/treaties/text.jsp?file_id=301019. $19 \mathrm{http}: / /$ bit.ly/2cCqD8m. 
Nutzung sollte über diese Schranke oder über eine andere neue Schranke explizit erlaubt werden. Aufgenommen werden sollte das Recht einer technischen Migration von einmal digitalisierten Werken.

\subsection{Regelung für den Umgang mit vergriffenen Werken durch Art. 7}

Maßnahme: Da für viele vergriffene Werke weiter Urheberrechtsschutz besteht, sieht es die EU-Kommission als notwendig an, den Ländern eine Schrankenregelung zugunsten der kulturbewahrenden Institutionen (Bibliotheken etc.) vorzuschlagen (,shall provide“). D.h. Bibliotheken bzw. allgemein „cultural heritage institutions“ soll es erlaubt sein, urheberrechtsgeschützte vergriffene Werke (nur) aus ihren eigenen Beständen zu digitalisieren, verbreiten und öffentlich zugänglich $\mathrm{zu}$ machen. Dies soll dann auch eine grenzüberschreitende Nutzung möglich machen. (vgl. EG 22-30)

Allerdings soll dies nur über Lizenzierungsvereinbarungen (bevorzugt „collective licensing“) mit den entsprechenden Verwertungsgesellschaften möglich sein. D.h. Kultureinrichtungen dürfen nicht von sich aus aktiv werden und ihre vergriffenen Werke digitalisieren, sondern nur dann ,when a collective management organisation, on behalf of its members, concludes a non-exclusive licence for non-commercial purposes with a cultural heritage institution“.

Die Verwertungsgesellschaften sollen (nicht-exlusive) Lizenzierungen auch von Werken solcher Autoren zuteilen dürfen, die nicht Mitglied der jeweiligen Verwertungsgesellschaft sind - allerdings dies nur unter der Bedingung, dass die Verwertungsgesellschaft für den betreffenden Werktyp eine repräsentative Anzahl von Autoren als ihre Mitglieder nachweisen kann. (Art. 7, 1,1)

Kritik: Eine Regelung für vergriffene Werke ist im Prinzip $\mathrm{zu}$ begrüßen. Jedoch ist dieser Artikel mit seinen fünf Absätzen und neun einschränkenden, untereinander verschachtelten Bedingungen so komplex und unverständlich angelegt, dass er kaum Klarheit für die Nutzung vergriffener Werke schaffen kann. Vermutlich ist diese Komplexität durch die Sorge begründet, dass diese neue Erlaubnis stark in die exklusiven Rechte von Autoren bzw. deren Erben eingreift. Allerdings bleibt diesen das Recht unbenommen, der Digitalisierung und der entsprechenden Nutzung jederzeit zu widersprechen.

Unklar ist, was geschehen soll, wenn der vorgesehene Widerspruch von Autoren erst dann erfolgt, wenn die Digitalisierung und Zugänglichmachung durch die Kulturein- richtungen schon erfolgt ist. Oder soll ihnen auferlegt werden, wie es bei der Regelung für verwaiste Werken geschehen ist, vor einer Digitalisierung durch eine sorgfältige Suche (,diligent search“) Kontakt zu den Rechtsinhabern (primär den Autoren) aufzunehmen und deren $\mathrm{Zu}$ stimmung einzuholen? Oder bleibt diese Vergewisserung in der Zuständigkeit der Verwertungsgesellschaften? In Art. 7 heißt es dazu nur lapidar: „Member states shall provide that appropriate publicity measures are taken ... including during a reasonable period of time before the works or other subject-matter are digitised, distributed, communicated to the public or made available“. Vielleicht deutet die Verwendung von „publicity measures“ darauf hin, dass keine „diligent search“ nötig sein muss, sondern das eine öffentliche (zeitlich befristete) Bekanntmachung des Vorhabens ausreicht. ${ }^{20}$

Ebenfalls ist nicht eindeutig geregelt, wie zu verfahren ist, wenn ein die Rechte besitzender Verlag das vergriffene Werke wieder neu und zwar auch digital auflegt. Muss dann die Nutzung des Werkes durch die Kultureinrichtungen eingestellt bzw. das Digitalisat sogar gelöscht werden?

Offensichtlich wird den Mitgliedsstaaten ein breiter Spielraum eingeräumt. Das kann auch wegen verschiedener kultureller Gepflogenheiten positiv sein, widerspricht aber dem angestrebten Ziel einer umfassenden Harmonisierung des Urheberrechts in der EU.

Ingesamt macht diese neue Schranke zugunsten der Digitalisierung und der Nutzung vergriffener Werke den Eindruck, dass sie nur unzureichend auf die Bedürfnisse und Besonderheiten von Kultureinrichtungen eingeht. So unterstützt z.B. der Deutsche Bibliotheksverband (dbv) diese Initiative im EU-Vorschlag im Prinzip, hat aber zahlreiche Verbesserungsvorschläge ${ }^{21}$, z. B. dass es kleineren Institutionen erlaubt sein soll, die Digitalisierung durch Dritte vornehmen zu lassen. Auch wird es nicht für angebracht gehalten, dass schon eine Übersetzung des vergriffenen Originalwerks dessen Digitalisierung und Nutzung aussetzt. Ebenso sollte die Nutzung vergriffener literarischer Vorlagen von Filmen erlaubt sein.

Vor allem aber wird es als Problem angesehen, dass den Verwertungsgesellschaften das ausschließliche Lizen-

20 Ausführlich dazu: Rainer Kuhlen: De revolutionibus orbium retiariorum - Besser mit dem Netz als suchen nach dem Matching-Paradigma - zur Legitimierung verwaister Werke - http://netethics.net/ netethicsblog/index.html\%3Fp=476.html.

21 Stellungnahme des Deutschen Bibliotheksverbandes (dbv): Entwurf der EU-Richtlinie zum Urheberrecht im digitalen Binnenmarkt vom 14. 9. 2016 (RL-E (COM (2016)593 final), zu Art. 7, S. 9 ff - http:// www.bibliotheksverband.de/fileadmin/user_upload/DBV/positionen/ 2016_10_28_dbv-Stellungnahme_EU-RL_Urheberrecht.pdf. 
zierungsrecht zugestanden wird. Der dbv fordert daher eine Art „Fallback“ -Möglichkeit für den Fall nicht zustande gekommener Vereinbarungen: Den Kultureinrichtungen sollte „die Nutzung vergriffener Werke im gesetzlich vorgesehenen Rahmen auch ohne diesen Gesamtvertrag gegen eine angemessene Lizenzgebühr erlaubt werden“. Für die erforderliche Vergütung sollten „objektive Kriterien wie z.B. der Vergütung vergleichbarer Nutzungen“" durch die Schranke vorgegeben werden.

\section{Weitere Defizite}

\subsection{Ausleihe und Versand digitaler Objekte durch Bibliotheken bleibt ausgeklammert}

Der Vorschlag der EU-Kommission geht mit Blick auf Bibliotheksleistungen lediglich auf das erwähnte Vervielfältigungsrecht zum Zwecke der Bewahrung von Objekten des kulturellen Erbes und den Umgang mit vergriffenen Werken ein.

Nicht geregelt ist das Recht auf den Erwerb von digitalen Objekten allgemein bzw. spezieller das Recht auf das Ausleihen solcher über Lizenz oder Kauf „erworbener“ digitaler Objekte. Der Europäische Gerichtshof hat Mitte November 2016 mit Verweis auf die EU-Richtlinie zum Vermiet- und Verleihrecht von 1992 in einer „dynamischen“ und ,evolutiven“ Auslegung (so Maciej Szpunar, Generalanwalt beim Europäischen Gerichtshof) entschieden, dass Bibliotheken das Ausleihen von E-Books nicht streitig gemacht werden kann. ${ }^{22}$ Dies ist auch für wissenschaftliche Bibliotheken wichtig, aber vor allem für öffentliche Bibliotheken unverzichtbar, wenn sie ihren öffentlichen Auftrag wahrnehmen wollen, ihre Kunden weiter mit aktuellen, oft nur noch in elektronischer Version vorliegenden Texten zu versorgen.

Die EU-Kommission sollte sich diese Rechtsprechung jetzt zu eigen machen und in ihrer Regelung darauf achten, dass die häufig noch DRM-gesteuerten Lizenz-Nutzungsbedingungen und die Vergütungsbestimmungen nicht zu restriktiv ausfallen. So wäre es nicht mehr zeitgemäß, auf das E-Lending das Prinzip der Bestandsakzessorietät anzuwenden. ${ }^{23}$ Ebenso sollte es nicht den Bibliotheken auf-

22 Vorabentscheidung des EuGH im Fall C 174/15 (Vereniging Openbare Bibliotheken v Stichting Leenrecht); vgl. https://irights.info/arti kel/eugh-openbare-bibliotheken-e-lending/28165.

23 „Bestandsakzessorietät“ bedeutet, dass weitere Ausleihvorgänge ausgesetzt werden, solange eine Ausleihe noch aktiv ist bzw. dass nur so viele Ausleihen simultan vorgenommen werden dürfen, wie es die jeweiligen Verlagsverträge vorsehen. gebürdet werden, für die DRM-Software zur Kontrolle der elektronischen Nutzung selber zuständig zu sein - z. B. für das automatische Löschen einer Datei beim Nutzer, wenn die Ausleihzeit abgelaufen ist.

Das Recht auf den Versand von digitalen Kopien an externe Nutzer bleibt in dem Vorschlag der EU-Kommission unbefriedigend ungeklärt. Es ist irritierend, dass nun, entsprechend des noch für gültig gehaltenen Erwägungsgrunds 40 aus Richtlinie 2001/29/EC RN 40, weiter gilt: „Eine Nutzung im Zusammenhang mit der Online-Lieferung von geschützten Werken oder sonstigen Schutzgegenständen sollte nicht unter diese Ausnahme fallen.“

Sofern Regelungen zum Kopienversand in den Regelungen der Mitgliedsstaaten bislang entsprechend InfoSoc 2001 umgesetzt sind, sind diese kaum noch zeitgemäß (z.B. Beschränkung auf Fax oder grafische, nicht durchsuchbare Dateien). Hier sollte die EU-Kommission ein deutliches Zeichen mit nutzerfreundlichen, den elektronischen Umgebungen angemessenen Regelungen setzen. Der dbv weist zudem darauf hin (vgl. FN 21), dass eine EUweite Harmonisierung auch des grenzüberschreitenden Kopienversands dringend erforderlich ist. Die Rechtslage ist in den verschiedenen Ländern so unübersichtlich, dass entsprechenden Anfragen zum elektronischen Kopienversand häufig nicht entsprochen werden kann. Sollte die Voll-Harmonisierung noch nicht möglich sein, „wäre den Bibliotheks-NutzerInnen mit einer Teilharmonisierung i.V. mit einer gegenseitigen Anerkennung der einschlägigen Normen - nach dem Vorbild der Regelung in Art. 4 Abs. 3 RLE geholfen. Dadurch würde der grenzüberschreitende Kopienversand rechtssicherer werden.“

\subsection{Senkung von Transaktionskosten wird nur angekündigt}

Die Absicht der Kommission, auch für Forschungseinrichtungen eine Reduktion der Transaktionskosten anzustreben, ist zu begrüßen. Wie das erreicht werden soll, bleibt allerdings offen, zumal - als offensichtlicher Erfolg des entsprechenden Lobbying - die Auswirkungen der Reduktion nicht zu Lasten der KMU in der Publikationswirtschaft gehen dürfen.

Weiter soll die Senkung der Kosten nicht durch Erweiterung offener/freier Nutzungsformen über rechtliche Schrankenregelungen erreicht werden, sondern durch Lizenzierungspraktiken (Abschnitt „Regulatory fitness and simplification").

Auch hier setzt die EU-Kommission keine Priorität zugunsten der offenen freizügigen Nutzung gegenüber der Sicherung von Verlagsinteressen und von elektronischen 
Umgebungen nicht mehr zeitgemäßen Geschäftsmodellen.

Die Reduktion von Transaktionskosten könnte in erster Linie durch die Einführung einer Allgemeinen Bildungs- und Wissenschaftsschranke erzielt werden (vgl. Abschnitt 2.4), aber auch durch eine Neuregelung der Vergütung im Wissenschaftsurheberrecht (Näheres dazu in Abschnitt 2.6).

\subsection{Allgemeine Bildungs- und Wissen- schaftsschranke (Comprehensive Clause for Education and Research - CCER)}

Obgleich in der Diskussion um ein wissenschaftsfreundliches Urheberrecht ${ }^{24}$ seit Jahren immer wieder betont wird, dass die Unzulänglichkeiten bei den auf Bildung und Wissenschaft bezogenen Schranken nicht durch kleinere Änderungen beseitigt werden können, bleibt in dem Vorschlag der Kommission die Realisierung einer umfassenden Allgemeinen Bildungs- und Wissenschaftsschranke (ABWS) ausgeklammert. Es ist nicht annehmbar, dass die EU-Kommission den Urheberrechtsproblemen für Wissenschaft (bis auf die neue TDM-Regelung) keine weitere Aufmerksamkeit geschenkt hat.

Hilfreich wäre es zumindest, wenn die bislang nur optionale Regelung für wissenschaftliche Forschung aus Richtlinie 2001/29/EC, Art. 5, 3, a nun rückwirkend für die Mitgliedsländer verpflichtend gemacht würde: „use for the sole purpose of illustration for teaching or scientific research, ... to the extent justified by the non-commercial purpose ..... Diese Formulierung sollte es in seiner Allgemeinheit erlauben, aus ihr direkt eine ABWS auf der jeweiligen nationalen Ebene abzuleiten.

Allerdings sind die bisherigen Umsetzungen dieses Schrankenvorschlags von 2001 sehr restriktiv ausgefallen - in Deutschland in $\S 52 \mathrm{a}$, aber auch in $\S 52 \mathrm{~b}$ und $\S 53 \mathrm{a}$ des deutschen Urheberrechtsgesetzes. Das liegt vor allem an der Orientierung an dem Dreistufentest, wie er in InfoSoc 2001 in Art. 5.5 explizit aufgegriffen und vorgegeben wurde: „The exceptions and limitations provided for in

24 Vgl. die Beiträge des Autors: Wie umfassend soll / darf / muss sie sein, die allgemeine Bildungs- und Wissenschaftsschranke? Zeitschrift für Geistiges Eigentum / Intellectual Property Journal, Volume 7, 2015, Number 1, pp. 77-125; Die Allgemeine Bildungs- und Wissenschaftsschranke im Urheberrecht nimmt Fahrt auf - Kreativität und Innovation werden die Gewinner sein. Information-Wissenschaft \& Praxis 2016; 67(1): 1-9; Der Heizer soll nicht auf der e-Lok bleiben - Die Allgemeine Bildungs- und Wissenschaftsschranke ist nötig und möglich. Zeitschrift für Urheber- und Medienrecht (ZUM) 6/2016, 507-513. paragraphs 1, 2, 3 and 4 shall only be applied in certain special cases which do not conflict with a normal exploitation of the work or other subject-matter and do not unreasonably prejudice the legitimate interests of the right holder."

Die aus dieser strikt interpretierten Vorgabe abgeleiteten Beschränkungen in den verschiedenen Schrankenbestimmungen (der Mitgliedsländer) sind dem heutigen digitalen Umfeld und der Praxis des Publizierens nicht mehr angemessen. ${ }^{25}$ Die EU-Kommission sollte sich nicht länger an einer rigiden Interpretation des Dreistufentests orientieren. Vor allem im Bereich von Wissenschaft (aber in absehbarer Zukunft auch immer mehr im Bildungsbereich über Open Educational Resources (OER)) wird die offene und freie Nutzung publizierten Wissens (Open Access) der allgemeine Trend und in vorhersehbarer Zukunft auch die durchgängige Praxis des wissenschaftlichen Publizierens sein - auch in der kommerziellen Informationswirtschaft. Dadurch würde der Dreistufentest auf allen drei Ebenen eine ganz andere Bedeutung bekommen: Die freie Nutzung ist die Regel - die proprietäre Nutzung die Ausnahme.

Trotz des erkennbaren Trends zugunsten des OpenAccess-Publizierens besteht weiter der Bedarf nach urheberrechtlichen Regelungen der Nutzung publizierter Materialien - nicht zuletzt auch wegen der Nicht-Open-AccessBestände aus der Vergangenheit, aber auch durch die Praxis der Verlage, vergriffene Werke zu digitalisieren und so neu auf den Markt zu bringen.

Dem sollte heute in einer umfassenden ABWS Rechnung getragen werden, welche die offene und freie Nutzung publizierten Wissens, vor allem des überwiegend mit öffentlichen Mitteln geförderten Wissens erlaubt. Diese Nutzung bezieht sich auf das Vervielfältigungsrecht und das Recht der öffentlichen Zugänglichmachung - vorgesehen für Mitglieder in Forschungsgruppen und für Teilnehmer an Ausbildungsveranstaltungen auf allen Bildungsebenen. Für diese Nutzung sollten keine Umfangsbeschränkungen vorgenommen werden. So wie schon in InfoSoc 2001 Art. 5, 3, a vorgesehen, soll der Zweck der jeweiligen Forschung und der jeweiligen Ausbildungsaktivität die ausreichende Legitimation für die Nutzung sein.

Eine umfassende Regelung für eine ABWS auf EUEbene könnte wie folgt aussehen. Dies ist allerdings kein ausformulierter Schrankenvorschlag, sondern soll nur den

25 Vgl. Eine ausgewogene Auslegung des Drei-Stufen-Tests im Urheberrecht (deutsche Übersetzung der „Declaration on a balanced interpretation of the „Three -StepTest“ in Copyright Law“) - http:// www.ip.mpg.de/fileadmin/ipmpg/content/forschung_aktuell/01_ba lanced/declaration_three_step_test_final_deutsch1.pdf. 
Rahmen einer europäischen Lösung für eine ABWS darstellen: ${ }^{26}$

(1) Erlaubt ist Vervielfältigung und öffentliche Zugänglichmachung von Werken für die Nutzung ausschließlich zur Veranschaulichung des Unterrichts (auf allen Bildungsebenen) oder für Zwecke der wissenschaftlichen Forschung in Forschungsgruppen, soweit dies zur Verfolgung nicht kommerzieller Zwecke gerechtfertigt ist.

(2) Satz 1 schließt auch Folgehandlungen wie für TDM mit ein.

(3) Der Zugriff auf Bildung und Forschung unterstützende Handlungen der Bibliotheken, einschließlich der Nutzung ihrer digitalisierten kulturbewahrenden Objekte und einschließlich der Folgehandlungen wie Abspeichern und Ausdrucken, ist vor Ort, aber auch durch externen, gesicherten, autorisierten Zugriff (z. B. über VPN) erlaubt.

(4) Die schrankenabhängigen Folgenutzungen von rechtmäßig erworbenen Werken für Zwecke der Ausbildung und von Forschung für nicht-kommerzielle Zwecke sind vergütungsfrei.

\subsection{Einführung eines Leistungsschutzrechts für Presseverleger nicht angebracht}

Es wird - auch nach den Erfahrungen in Deutschland mit einer entsprechenden Regelung - für einen Fehler und für eine ernsthafte Einschränkung von Link-/Informationsfreiheit gehalten, dass die EU-Kommission über Art. 11 ein Leistungsschutzrecht für Presseverleger (nicht allgemein für Verleger) einführen will. Dieses Leistungsschutzrecht betrifft mit negativen Folgen auch Bildung und Wissenschaft. In manchen Sektoren können mediale Objekte jeder Art der Presse durchaus auch Gegenstand von Forschung und vor allem auch von Lehre und Lernen der Ausund Weiterbildung auf allen Ebenen sein.

Das in Deutschland schon eingeführte Leistungsschutzrecht für Presseverleger hat sich (so die überwiegende Einschätzung der Fachwelt außerhalb der Presselobby selber) als offensichtlicher Fehlschlag herausgestellt. Nicht zuletzt haben sich weder die Einnahmen der Verlage dadurch vergrößert, noch konnten die Journalisten als Autoren daraus Nutzen ziehen.

Die Reaktionen auf die beabsichtigte Einführung eines neuen EU-Rechts (Leistungsschutzrechts) in Art. 11 sind

26 Konkrete Vorschläge liegen in Deutschland vor u.a. vom Aktionsbündnis „Urheberrecht für Bildung und Wissenschaft“ http://www. urheberrechtsbuendnis.de/abws-text-2014-12.html.de sowie, im Auftrag des deutschen Forschungsministeriums, von Prof. Dr. Katharina de la Durantye http://durantaye.rewi.hu/doc/Wissenschaftsschra nke.pdf. durchweg negativ. In einer Erklärung von 30 Rechtswissenschaftlern im Vereinigten Königreich heißt es: „we believe the proposed right is unnecessary, undesirable, would introduce an unacceptable level of uncertainty and be unlikely to achieve anything apart from adding to the complexity and cost of operating in the copyright environment“. ${ }^{27}$ Auch der BDI (vgl., die Spitzenorganisation der deutschen Industrie und der industrienahen Dienstleister, ,warnt davor, den Fehler der digital- und ordnungspolitisch schädlichen und letztlich auch für die deutschen Presseverlage erfolglosen Einführung eines solchen Rechts nun auf EU-Ebene zu wiederholen, und lehnt die Einführung eines Leistungsschutzrechts für Presseverleger in vollem Umfang ab. Andernfalls drohen jahrelange Rechtsunsicherheiten, unkalkulierbare Lizenzierungspflichten für zahlreiche deutsche (Industrie-)Unternehmen und die Verhinderung neuer, innovativer Geschäftsmodelle im Internet. Zudem überzeugen die Argumente für ein Leistungsschutzrecht auch in der Sache nicht. “'28

Wenn überhaupt etwas zugunsten der Presseverlage getan werden soll, dann wäre das Mittel nicht, ein neues Recht einzuführen, sondern die Lizenzierungsmöglichkeiten auszunutzen oder gar von den Medien selber entsprechende Dienste zu entwickeln, durch die ein Teil des Internet-Verkehrs, der bislang überwiegend aus dem Nachweis der Presseerzeugnisse durch Suchmaschinen resultiert, an die Presseverlage direkt gehen könnte.

In Deutschland wird vor allem auf der Website IGEL ${ }^{29}$ (Initiative gegen ein Leistungsschutzrecht) gegen das (in Deutschland schon eingeführte und jetzt in der EU geplante Leistungsschutzrecht für Presseverleger) opponiert. Es werden zudem Vorschläge gemacht, wie Verbesserungen zugunsten der Verlege ohne die Einführung eines neuen Rechts erreicht werden können. ${ }^{30}$ IGEL bringt diese Argumente auch über c4c (Copyright for Creativity) ${ }^{31}$ in die europäische Diskussion ein.

27 http://www.iposgoode.ca/wp-content/uploads/2016/12/IPOMod ernisingIPProfResponsePressPublishers.pdf.

28 https://www.bmjv.de/SharedDocs/Gesetzgebungsverfahren/Stell ungnahmen/2016/Downloads/11112016_Stellungnahme_BDI_EU-Urh eberrechtsreform.pdf?_blob=publicationFile \&v=2.

$29 \mathrm{http}: / /$ leistungsschutzrecht.info/.

$30 \mathrm{http} / / /$ ancillarycopyright.eu/news/2016-11-23/how-solve-only-spe cific-problem-press-publishers-copyright-without-ancillary-copyright. 31 http://copyright4creativity.eu/. 


\subsection{Regelung der Beteiligung der Verlage an den Ausschüttungen der Verwertungsgesellschaften}

Die jüngsten hier einschlägigen Entscheidungen sowohl des EuGH als auch des $\mathrm{BGH}^{32}$ hatten klargestellt, dass es die Autorinnen und Autoren sind, die als die originalen Rechtsinhaber zu gelten haben, und dass daher ihnen und nicht den Verlegern als den Inhabern sekundärer Nutzungsrechte die volle Vergütung durch die Verwertungsgesellschaften für die Nutzung ihrer Werke zusteht. Entsprechend dürfen nun die Verlage in Ländern, in denen, wie in Deutschland über die VG-Wort, aber auch über die GEMA und VG BildKunst, ein Teil der Ausschüttungen der Verwertungsgesellschaften an die Verlage ging, nicht mehr daran beteiligt werden. Sie müssen sogar rückwirkend für einige Jahren die ihnen nicht zustehenden Beträge wieder zurückzahlen.

Es ist nicht leicht nachzuvollziehen, warum die EUKommission diese Entscheidungen der obersten Gerichte ignoriert, indem sie in Art. 12 (Claims to fair compensation) die Beteiligung der Verleger an der Ausschüttung der Verwertungsgesellschaften nun rechtlich als „fair compensation“ regeln will (vgl. auch Abschnitt 2.7 zur Vergütungsfrage im Bereich Wissenschaft.). Dies ist nicht nur eine Benachteiligung der Autoren und Autorinnen, sondern m. E. auch eine wirtschaftspolitisch falsche Entscheidung.

Zudem scheint es mir urheberrechtssystematisch problematisch zu sein, aus der über Verträge erfolgten Übertragung der Verwertungsrechte der Urheber als Nutzungsrechte an die Verleger zu folgern, dass nun auch den Verlegern Anteile an der durch Schrankenregelungen entstandenen Nutzungen zuzugestehen ist. Schranken werden ausschließlich durch das öffentliche Interesse an einer genehmigungsfreien Nutzung urheberrechtsgeschützter Werke legitimiert. Verlage sichern ihre finanzielle Basis durch Verkauf oder Lizenz der ihnen von den Autoren bereitgestellten Werke ab. Weitere Einnahmen über Schrankennutzungen sollten nicht über das Urheberrecht garantiert werden. Finanzierungsprobleme kommerzielle Verwerter sollten nicht über das Urheberrecht gelöst werden sollen. Durch Vorschläge wie in Art. 12 werden Verlage ermuntert, an ihren alten und in elektronischen Umgebungen obsoleten Publikations-/Geschäftsmodellen festzuhalten, anstatt innovative Verfahren (Produkte und Dienstleistungen) auf den Markt zu bringen, für die dann

32 „Reprobel“-Urteil des EuGH vom 12. November 2015 in der Rechtssache C-572/13; darauf folgend das „Vogel“-Urteil des BGH vom 21. April 2016, ZR 198/13. Zu beiden Urteilen vgl. ausführlich: https:// irights.info/artikel/eugh-reprobel-vogel-vgwort-c-572-13/26429. auch Einnahmen (nicht zuletzt auch durch Web-Traffic) erzielt werden können. (vgl. Abschnitt 2.4)

Allerdings überwiegt derzeit in der europäischen, aber auch in der deutschen Politik die Ansicht, dass das jahrelang praktizierte Verfahren, Verleger an der Ausschüttung der Verwertungsgesellschaften zu beteiligen, fair und angemessen gewesen sei und nun juristisch wasserfest gemacht werden sollte.

In Deutschland wird als (gesetzlich geregelte) Übergangslösung versucht, den Verlagen dadurch entgegenzukommen, dass es den Autoren nach der Veröffentlichung eines verlegten Werkes oder mit der Anmeldung des Werkes bei der Verwertungsgesellschaft gestattet ist, auf den ihnen jetzt zustehenden Verlageranteil bei der Vergütung zugunsten der Verleger zu verzichten. ${ }^{33}$

Entsprechende Aufforderungen gingen seit Anfang 2017 von vielen Verlagen an ihre Autoren heraus. Dies ist eine (wohl bis Ende Februar) befristete Aktion. In welchem Umfang Autoren sich darauf eingelassen haben, wurde bislang nicht öffentlich gemacht. Den jeweiligen nationalen Gesetzgebungen war es wegen der derzeit noch existierenden unionsrechtlichen Vorgaben nicht möglich, eine weitergehende Lösung zugunsten der Verlage zu erreichen. Dafür müssen erst in der EU die Voraussetzungen, wie jetzt in Art. 12 des Kommissionsvorschlags geplant, geschaffen werden. Ob und wann das geschieht, ist offen. Bis dahin haben die Verlage keinen direkten Anspruch auf eine Beteiligung an den Ausschüttungen der Verwertungsgesellschaften.

\subsection{Vergütung im Bereich Bildung und Wissenschaft}

Die Vergütungsfrage wird im Vorschlag der EU-Kommission kaum aus der Perspektive von Bildung und Wissenschaft angesprochen. Diese sollte in den Wissenschaftsund Bildungsmärkten ganz anders beantwortet werden als auf den allgemeinen Publikumsmärkten. Dazu nur einige Hinweise:

1. Die weitaus überwiegende Mehrheit der Wissenschaftler ist an einer monetären Anerkennung ihrer Arbeit nicht interessiert. Wie jüngst noch einmal durch eine von der EU in Auftrag gegebene Studie bestätigt wird, ${ }^{34}$

33 Vgl. dazu die Sicht der VG-Wort - https://www.boersenblatt.net/ar tikel-interview_mit_robert_staats_geschaeftsfuehrer_der_vg_wort.12 66695.html; der Autoren-Musterbrief des Börsenvereins unter: https:// www.pdf-archive.com/2016/11/06/boev-info-vg-wort-okt-2016/. 34 Europe Economics; Lucie Guibault and Olivia Salamanca of the University of Amsterdam: Remuneration of authors of books and 
erhalten Autoren in dem für Wissenschaft wichtigsten Sektor - Artikel in wissenschaftlichen Zeitschriften so gut wie nie eine Vergütung von den Verlagen (bis auf wenige Buchausnahmen und Fachgebiete wie Jura), denen sie ihre Verwertungsrechte als Nutzungsrechte per Vertrag abgetreten haben.

2. Schrankenbezogene Nutzung sollte von solchen Werken nicht vergütungspflichtig sein, die überwiegend durch Autoren entstanden sind, die öffentlich finanziert werden. Dieser Gruppe der Autoren - und das ist der weitaus größte Teil aller Autoren nicht nur der Hochschulen, sondern auch der Forschungsinstitutionen außerhalb der Hochschulen, einschließlich der Ressortforschungen der Ministerien - sollte nicht als Spezialfall, sondern als Regelfall Rechnung getragen werden.

3. Auch in der EU sollten zukunftsweisende Überlegung angestellt werden, „das Urheberrecht dahingehend zu ändern, dass urheberrechtlich geschützte Werke unentgeltlich im Schulunterricht sowie an Universitäten genutzt werden können. “ Das ist keine realitätsfremde Forderung. Das Zitat stammt aus dem Petitionssauschuss des Deutschen Bundestags, der einstimmig am 25. Februar 2015 empfohlen hat, ${ }^{35}$ solche Überlegungen bei der Neugestaltung des Urheberrechts mit einzubeziehen.

4. Auch Art. 4, 4 des Vorschlags der EU-Kommission von 2016 schreibt für „digital and cross-border teaching activities" Vergütung nicht verpflichtend vor. Es wird nicht von „shall“ gesprochen, sondern von „may provide for fair compensation“. Auch der Vorschlag der EU-Kommission für eine neue Schranke für Text and Data Mining (TDM) sieht keine Entschädigung (Vergütung) vor, weil der Schaden für die Rechtsinhaber durch TDM-Nutzung minimal sei. (vgl. EG 13). Dieses Argument sollte für die schrankenabhängige Nutzung in Bildung und Wissenschaft allgemein verwendet werden.

Urheberrechtsregulierungen und entsprechende Rechtsprechungen sollten diese Tatsachen berücksichtigen und

scientific journals, translators, journalists and visual artists for the use of their works. 2016 - Volltext: http://ec.europa.eu/newsroom/ dae/document.cfm?action=display\&doc_id=17026r.

35 https://www.bundestag.de/presse/hib/2015_02/-/362606. Die dem zugrunde liegende Petition bezog sich auf Mitschnitte im TV-Programm durch Schüler und Studenten. Obwohl diese Petition die 50.000 Unterzeichner-Hürde sehr deutlich nicht überspringen konnte, hat der Petitionsausschuss die Frage aufgegriffen und für „urheberrechtlich geschützte Werke“ insgesamt verallgemeinert. nicht undifferenziert das Recht auf Vergütung von Nutzungen auf Grund von Schrankenerlaubnisse anfordern. Im Wissenschaftsbereich ist die Währung Reputation, nicht finanzielle Remuneration.

Eine Neuregelung der Vergütung im Wissenschaftsurheberrecht sollte vor allem in der einzurichtenden Allgemeinen Bildungs- und Wissenschaftsschranke (ABWS) bzw. in vergleichbaren Verbesserungen des Wissenschaftsurheberrechts ihren Niederschlag finden.

\section{Fazit}

Es ist der EU-Kommission trotz einiger positiver Weiterentwicklungen nicht gelungen, einen der gegenwärtigen Praxis der Produktion und Nutzung von Wissen und Information gerecht werdenden Vorschlag oder gar einen in die Zukunft weisenden Paradigmenwechsel für die Urheberrechtsregulierung vorzulegen.

Der schwerwiegendste Einwand gegen den Vorschlag der EU-Kommission, eine neue Urheberrechtsrichtlinie in der vorgelegten Fassung auf den Weg zu bringen, besteht darin, dass die EU-Kommission weiter an dem alten Paradigma festhält, dass das Urheberrecht in erster Linie dazu dienen soll, die europäischen Binnenmärkte für Wissen und Information nicht nur funktionsfähig zu halten, sondern ihren weiteren Ausbau durch Festhalten an alten Geschäftsmodellen zu unterstützen.

Diese Funktionalisierung des Urheberrechts muss, wie zu Anfang gesagt, nicht unbedingt kritisiert werden - aber nur dann nicht, wenn unter „Märkten“ nicht nur die kommerziellen, auf den Schutz proprietärer Eigentumsrechte pochenden Verwertungsformen verstanden würden, sondern auch die offenen, auf freien Zugang zu und freie Nutzung von Wissen und Information setzenden Austauschformen eingeschlossen werden. Letzteres ist allerdings kaum erkennbar.

Ein defensives, auf den Schutz bestehender Geschäftsmodelle für die Verwertung von Wissen und Information setzendes Verständnis von Urheberrecht lag der bis heute noch gültigen Urheberrechtsrichtlinie von 2001 zugrunde. Leider war die EU-Kommission offenbar nicht in der Lage oder auch nicht willens, sich von diesem Verständnis zu lösen. Ganz im Gegenteil - weiter steht im Vordergrund der Schutz der Rechte der traditionellen Verwertungsunternehmen. Bezeichnend dafür sind u. a. die Art. 11 und 12 des Vorschlags der EU-Kommission.

Der jetzige Vorschlag für eine Copyright-Richtlinie muss jedoch nicht das letzte Wort der EU sein. EU-Parlament und EU-Rat müssen dem Vorhaben zustimmen. Vor allem das Parlament, das sich trotz mannigfacher Ein- 
schränkungen dann doch den Reda-Vorschlag zu eigen gemacht hat, ${ }^{36}$ wird hoffentlich nicht einknicken. Es sollte, wie im Reda-Bericht gefordert, ein offeneres, zeitgemäßes, den digitalen Rahmenbedingungen Rechnung tragendes Urheberrecht einfordern.

In dem Verfahren ist vorgesehen, dass zunächst die einschlägigen Komitees des EU-Parlaments zu dem Entwurf des EU-Kommission Stellung nehmen. Für das jetzige Vorhaben ist das JURI-Committee (Legal affairs) federführend, begleitend/beratend aber auch INTA (International Trade), ITRE (Industry, Research and Energy), IMCO (Internal Market and Consumer Protection) und CULT (Culture and Education). Für die Beratungen der Komitees können im Prinzip von jedermann sogenannte Draft opinions eingereicht werden. ${ }^{37}$

Auch der EU-Rat, das Ensemble der Chefs der nationalen Regierungen, kann und sollte mehr verlangen, als die EU-Kommission nun vorgelegt hat. Immerhin hat sich der Rat Mitte Mai 2016 einem Konzept für ein „open science system" angeschlossen, ${ }^{38}$ das anlässlich der niederländischen EU-Rats-Präsidentschaft im April 2016 auf einer Konferenz „On Open Science - From Vision to Action“ erarbeitet und verabschiedet wurde.

- Das Engagement der beiden niederländischen Präsidenten im EU-Rat, Henk Kamp und Sander Dekker für Open Science zeigt deutlich, wie stark nationale Initiativen auf die EU-Politik einwirken können.

- Auch dem Vorschlag für TDM im vorliegenden Kommissionsentwurf ist die Verabschiedung einer entsprechenden Schrankenbestimmung im UK-Copyright von Juni 2014 vorangegangen. Hier hat sich Großbritannien erneut nicht gescheut, entgegen der immer noch gültigen InfoSoc-Richtlinie von 2001, eine ganz neue Schrankenbestimmung zu verabschieden.

- Auch die deutsche Regelung für die vergriffenen Werke (in Ergänzung zu der zu den verwaisten Werken) hat vermutlich für die EU-Kommission einen wichtigen Anstoß gegeben - was sich in den Art. 7 und 8 niedergeschlagen hat.

Es ist zu hoffen und wird nicht zuletzt aus der Sicht von Bildung und Wissenschaft erwartet, dass die EU-Kommission sich nach den Beratungen im Parlament und entsprechend aktueller Entwicklungen und Wünsche in den Mitgliedsländern entschließt, einen neuen Vorschlag für eine

36 http://bit.ly/2cgKWZ6.

37 Draft opinions für JURI unter http://www.europarl.europa.eu/ committees/en/juri/draft-opinions.html und für CULT unter: http:// www.europarl.europa.eu/committees/en/cult/draft-opinions.html. $38 \mathrm{http} / / /$ bit.ly/2944rFa.
Urheberrechts-Richtlinie vorzulegen, der dem neuen Paradigma einer offenen und freien Nutzung von Wissen und Information besser Rechnung trägt, ohne dabei die innovative Weiterentwicklung der kommerziellen Informationswirtschaft zu behindern oder gar auszuschließen. Es ist allerdings kaum zu erwarten, dass der politische Meinungsbildungsprozess in der EU noch im Jahr 2017 abgeschlossen sein wird.

Auf die aktuellen Bemühungen in Deutschland, das Urheberrecht, vor allem mit Blick auf das Wissenschaftsurheberrecht, an die stark veränderten technologischen, ökonomischen und informationsethischen Rahmenbedingungen des Umgangs mit Wissen und Information anzupassen, wird in einem zweiten Beitrag näher eingegangen. Hier stand lange die Auseinandersetzung um eine Allgemeine Bildungs- und Wissenschaftsschranke (ABWS) im Vordergrund. Diese Forderung steht nach wie vor im Raum, obgleich der Ende 2016 öffentlich gemacht Vorschlag des BMJV keine ABWS, sondern acht neue Schrankenregelungen vorsieht, durch die die gravierendsten Probleme bei den bisherigen Schrankenregelungen (vor allem in den $\S \S 52 a, 52 b$ und 53a) gelöst werden sollen. Dieser derzeit heftig diskutierte Vorschlag beruht noch auf den derzeit gültigen unionsrechtlichen Vorgaben und konnte bzw. wollte noch nicht die zum Teil weitergehenden Vorhaben der EU-Kommission (vgl. Kap. 1) berücksichtigen. Auch hier ist es offen, ob über diese Initiative noch in der laufenden Legislaturperiode im Bundestag abschließend beraten werden kann.

Mit einem zugleich weinenden und lachenden Auge kann daher nicht ausgeschlossen werden, dass ab 2018 sowohl in der EU als auch in Deutschland die Karten ganz neu gemischt werden.

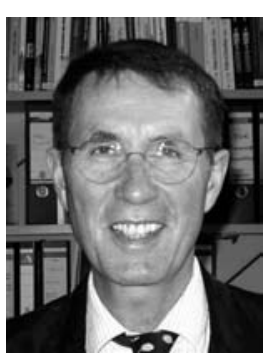

Prof.-em. Dr. Rainer Kuhlen

Bogotastraße 4

14163 Berlin

www.kuhlen.name

Blog: www.netethics.net

ORCID: 0000-0002-4497-6422

rainer.kuhlen@uni-konstanz.de

Prof.-em. Dr. Rainer Kuhlen ist emeritierter Professor für Informationswissenschaft im Fachbereich Informatik und Informationswissenschaft an der Universität Konstanz und Sprecher des Aktionsbündnisses „Urheberrecht für Bildung und Wissenschaft“. 